\title{
What Sonic did next
}

The signalling molecule Sonic hedgehog (Shh) has been investigated so extensively over the last few years that it is a wonder that there is anything left to discover about its properties. However, as Charron and colleagues show in a report in Cell, it still has a few surprises up its sleeve. In addition to its classic role as a morphogen, it can also act as an axon guidance cue.

In the vertebrate embryo, a group of neurons that originate in the dorsal spinal cord send axonal projections to the ventral midline (or floor plate), where they cross to the other side to generate commissural tracts. Netrin 1 has been identified as an essential chemoattractant that guides these axons towards the ventral midline. However, it cannot be the only factor that is required, because in Netrin 1 knockout mice, some of these axons are still attracted towards the floor plate.

As Shh is expressed in the floor plate of the vertebrate neural tube, and can act at a long range from its source, it was considered to be a possible candidate for one of the missing factors. Indeed, Charron et al. showed that Shh, like Netrin 1, can reorientate commissural axons in vitro. They also showed that the growth cones of frog spinal axons could change their course and grow towards a source of $\mathrm{Shh}$, further supporting the idea that Shh acts directly as a chemoattractant.

By blocking the function of the Shh receptor Smoothened (Smo) using the specific inhibitor cyclopamine, the authors also obtained insights into the molecular mechanism that underlies the chemoattractant properties of Shh. They found that, at least in vitro, cyclopamine prevented commissural axons from turning towards a source of Shh, but did not affect their response to Netrin 1 . To find out whether Shh is required in vivo for commissural axon guidance, they selectively disrupted Smo function in commissural axons. Consistent with Shh acting as a midline chemoattractant, this resulted in defects in commissural axon guidance. Importantly, this also implies that the commissural neurons have a cell-autonomous requirement for Shh signalling.

So, Charron et al. provide compelling evidence that Shh, acting through Smo, functions as an axon guidance cue at the floor plate of the developing spinal cord. Unlike Netrin 1, it cannot promote axonal outgrowth, but it seems to be an essential component of the molecular mechanism that mediates attraction of axons to the ventral midline. These findings should stimulate investigations into whether other morphogens can 'double up' as axon guidance molecules once their patterning work is done.

Heather Wood

\section{(2) References and links} ORIGINAL RESEARCH PAPER Charron, F. et al The morphogen Sonic hedgehog is an axonal chemoattractant that collaborates with Netrin-1 in midline axon quidance. Cell 18 March 2003 (doi:10.1016/S0092867403001995)

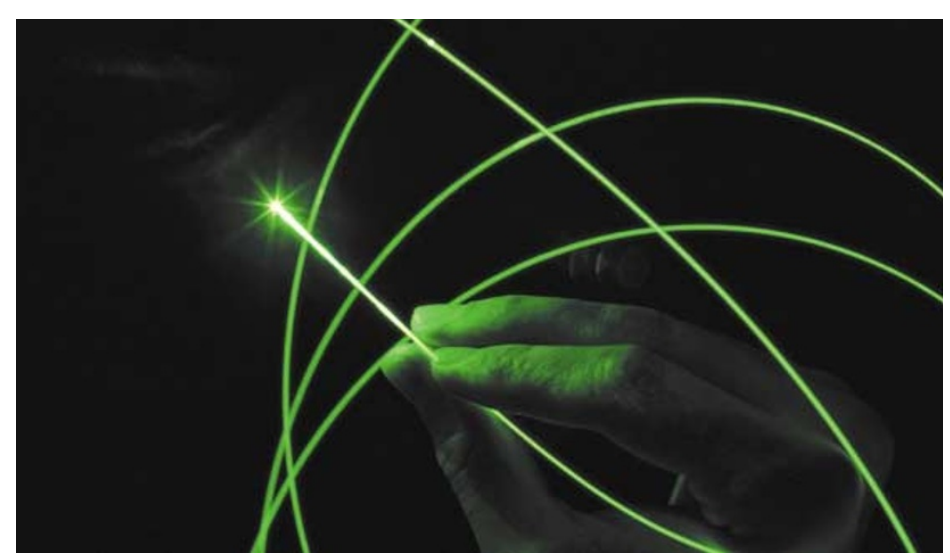

\section{IN BRIEF}

\section{SYNAPTIC TRANSMISSION}

Control of neurotransmitter release by an internal gel matrix in synaptic vesicles.

Reigada, D. et al. Proc. Natl Acad. Sci. USA 100, 3485-3490 (2003)

In neurotransmitter vesicles from Torpedo, $95 \%$ of acetylcholine and ATP is found to be adsorbed to an intravesicular proteoglycan matrix, rather than being in free solution. This 'smart gel', which includes the proteoglycan SV2, releases neurotransmitter in response to changes in ion concentration, and could modulate the amount of transmitter released during vesicle fusion.

\section{NEURODEGENERATION (}

Parkin suppresses dopaminergic neuron-selective neurotoxicity induced by Pael-R in Drosophila.

Yang, Y. et al. Neuron 37, 911-924 (2003)

Mutations in the parkin gene that abolish the protein's E3 ubiquitin ligase activity are associated with early-onset Parkinson's disease. One substrate of Parkin is Pael-R, and Yang et al. show that expression of human PAELR in Drosophila causes degeneration of dopamine neurons, and that coexpression of Parkin prevents this degeneration.

\section{CELL BIOLOGY OF THE NEURON}

The role of presenilin cofactors in the $\gamma$-secretase complex.

Takasugi, N. et al. Nature 422, 438-441 (2003)

Reconstitution of $\gamma$-secretase activity.

Edbauer, D. Nature Cell Biol. 7 April 2003 (10.1038/ncb960)

These two papers provide new insights into the components of $\gamma$-secretase, an unusual protease that is involved in the formation of amyloid- $\beta$ peptide by cleavage of amyloid precursor protein. Edbauer et al. show that $\gamma$-secretase activity can be reconstituted in yeast by the co-expression of human presenilin and three putative cofactors - nicastrin, APH1 and PEN2. Takasugi et al., meanwhile, have found evidence that, in Drosophila, APH1 stabilises the presenilin holoprotein in the complex formed by the proteins. PEN2 then seems to interact with the APH1-presenilin-nicastrin complex to cause endoproteolysis of presenilin and confer $\gamma$-secretase activity on the complex.

\section{DEVELOPMENT}

Wnt-mediated axon guidance via the Drosophila Derailed receptor

Yoshikawa, S. et al. Nature 16 March 2003 (10.1038/nature01522)

In Drosophila, axons that cross the midline during development through the anterior commissure express an atypical tyrosine kinase receptor, Derailed, that prevents them from entering the posterior commissure. Yoshikawa et al. show that Derailed mediates this repulsion by acting as a receptor for Wnt 5 , a secreted signalling molecule, and that Wnt 5 is expressed by neurons around the posterior commissure. 